\title{
Reply to: Observed impacts of the COVID-19 pandemic on global trade
}

\author{
Daoping Wang $\oplus^{1}$, Klaus Hubacek $\oplus^{2}$, Xi Liang $\oplus^{3}$, D'Maris Coffman $\odot^{4}$, Stephane Hallegatte $\oplus^{5}$ and \\ Dabo Guan $\oplus^{4,6} \bowtie$
}

REPLYING TO J. Verschuur et al. Nature Human Behaviour https://doi.org/10.1038/s41562-021-01060-5 (2021)

In Guan et al. ${ }^{1}$, we analysed the supply-chain effects of a set of stylized coronavirus disease 2019 (COVID-19) lockdown scenarios using a disaster assessment model ${ }^{2,3}$ in combination with a widely used and validated global trade database ${ }^{4}$. Despite considerable data limitations at this early stage of the outbreak, we provided a number of insights important for decision-makers attempting to minimize economic loss and acting under substantial urgency and uncertainty. In their recent communication, J. Verschuur and colleagues estimated global supply-chain dynamics using real-time ship tracking data and voiced some concerns on our modelling study ${ }^{1}$ based on the comparison of their findings with our modelling results. While we appreciate their complementary follow-up research based on real-time data, we posit that it is not possible to compare the stylized lockdown scenarios we developed with their data reflecting actual changes in the economy and policies that were implemented in response to COVID-19. In addition, there are differences in the modelling scope and their data has limitations with respect to how well it reflects changes in global supply chains. We call for broad and practical collaborations that integrate epidemiological modelling with economic forecasting tools to provide full information to both policy and public stakeholders about effective protective measures that are needed for public health as well as the economy.

First, in Guan et al. ${ }^{1}$ we did not aim to predict the true cost of COVID-19; instead, we illustrated possible consequences under different types of lockdown scenarios, none of which has been imposed exactly as described in any of the countries. It would have been impossible to assess the real costs at this early stage given the differences (and day-to-day adjustments) in actual responses across the globe. Forecasting the economic impact of the pandemic was impossible given the dynamics of the situation. Instead, we identified the most important aspects of disease control (such as strictness, duration and recurrence of lockdowns) by comparing the losses under several sets of idealized scenarios for containment measures and tested the sensitivity of these factors as their impacts ripple through global supply chains. The combinations of strictness and duration are based on stylized scenarios rather than the actual policies implemented in any particular country. Thus, a comparison of our findings based on types of lockdown measures with real-time shipping data reflecting changes in actual lockdown measures does not in any way assess the validity of our model and approach, as the underlying lockdown measures are not the same. While using real-time data is important and complementary to our modelling approach, it is not suited for validation of the model, given the differences of measures in the real world and those used in the scenarios. Instead, validation of the model would require input of precise country-by-country lockdown data, including data on trade and shipping restrictions, and comparing the model output with observed GDP losses.

Second, the data presented by Verschuur and colleagues ${ }^{5}$ reflect only a specific part of international trade, again making their results not comparable with those of our model, which considers all international trade. Although Verschuur and colleagues ${ }^{5}$ argue that "Maritime trade accounts for approximately $80 \%$ of international trade in terms of volume", about $40 \%$ of trade by value is dispatched by air ${ }^{6}$. Considering the different impacts of COVID-19 control measures on air and sea cargo ${ }^{7,8}$, it is difficult to use the estimated results from ship tracking data to represent changes in all international trade due to the pandemic. Change in online services is another factor that is included by Guan et al. ${ }^{1}$ but not by Verschuur and colleagues ${ }^{5}$. During the COVID-19 pandemic, online services related to physical production have declined due to the decline in physical production, whereas purely online services (such as education or meeting software services) have increased ${ }^{9}$.

The changes in the structure of trade structure under the impact of the COVID-19 pandemic have further contributed to uncertainty about the estimates of trade value. Verschuur and colleagues $^{5}$ established a conversion table describing the probability that a certain vessel type is associated with an economic sector using historical data. However, the rapidly changing trade structure during the pandemic ${ }^{10,11}$ is likely to affect their estimated probabilities. This may be the most important reason for the inconsistency between their estimates of disruption dynamics and the data released by the General Administration of Customs of Vietnam (see the paragraph below where we respond to their first concern). Moreover, it is very difficult to predict the load of each container or ship. Many fully filled containers are shipped from China to the EU and the United States. Some of these containers are sold and used within the EU and the United States, and cargo ships return with fewer containers or many empty containers. Thus, as can be seen from their estimates, the accuracy in predicting Japanese trade data by volume (supplementary fig. $1 \mathrm{~h}$ in Guan et al.) and export trade data by value (supplementary fig. $2 b$ in Guan et al.) is limited, especially for countries with low trade value. Customs trade data after the crisis will be a useful source for analysis of changes in trading patterns during the pandemic and subsequent recovery.

'School of Urban and Regional Science, Shanghai University of Finance and Economics, Shanghai, China. ${ }^{2}$ Integrated Research on Energy, Environment and Society (IREES), University of Groningen, Groningen, the Netherlands. ${ }^{3}$ Business School, University of Edinburgh, Edinburgh, UK. ${ }^{4}$ The Bartlett School of Construction and Project Management, University College London, London, UK. ${ }^{5}$ The World Bank, Washington, DC, USA. ${ }^{6}$ Department of Earth System Science, Tsinghua University, Beijing, China. 凶e-mail: guandabo@tsinghua.edu.cn 
In addition to the above points, we would like to address their concerns individually. Regarding their concerns about Vietnam: fig. 2 in Guan et al. ${ }^{1}$ shows that Vietnam would suffer about 5-30\% of value-added loss under different scenarios. The latest report from the General Department of Vietnam Customs shows that Vietnam's total imports in the first half of 2020 fell by $2.9 \%$ and total exports remained almost unchanged compared with 2019 $9^{10,11}$ (note that the global lockdown scenarios that we set were more stringent than those applied in reality, so the modelled impacts were larger than the actual impacts). Nevertheless, there have been increases in trading volumes in Vietnam and China in the last quarter of 2020 due to the failure of effective disease control leading to multiple lockdowns and delaying economic restarts in the West.

Regarding their concerns about en route goods: Verschuur and colleagues $^{5}$ argue that the en route goods serve as a positive buffer to the downstream sectors and slow the forward effect. Guan et al. ${ }^{1}$ modelled this aspect as the inventory of each sector, although this is a different concept to the idea of en route goods. Inventory would have a buffer role in reducing or delaying any forward propagation of shocks (illustrated by $\mathrm{CN}$ in supplementary fig. 2 in Guan et al. ${ }^{1}$ ). However, production is restricted by a number of factors, including raw material. During lockdown, limited labour availability, rather than raw material shortages, has usually been the key factor in rapid production declines (GB in supplementary fig. 2 in Guan et al. ${ }^{1}$ ). Therefore, the steeper decline in our simulation was because we considered factors other than trade.

Regarding their third concern, Verschuur and colleagues ${ }^{5}$ argue that heterogeneities exist by comparing import and export dynamics of countries with similar stringency dynamics of control measures. However, the stringency index that they present for the United States and the UK from April to July 2020 is almost same as the one for China in March (fig. 1c in Verschuur et al. ${ }^{5}$ ). However, anecdotal evidence suggests that the lockdown in China was much more stringent than any of the lockdowns in the UK or the United States, raising doubt about the usability of the stringency index for measuring the impact of the labour supply.

Overall, Verschuur and colleagues ${ }^{5}$ provide an interesting data-driven approach to monitor aspects of actual international trade flows, even though their study cannot offer a direct validation or further information on our modelling results. Both model-based and data-based approaches are needed to inform policymakers. For a more in-depth perspective, it is useful to borrow ideas from mixed-frequency modelling strategies to integrate high-frequency data into a disaster impact assessment system.

Finally, the main qualitative conclusions of our paper appear more valid than ever. First, short and intensive lockdowns that can to stop the epidemic appear less economically costly than less stringent lockdowns that slow down the spread without eliminating the disease. Second, overcoming the pandemic is a global public good. The end of the pandemic depends on the last group of countries that effectively implement protective measures, as well as international collaboration. Support made available to the poorest countries will be essential to minimize the duration and economic cost of the pandemic.

Received: 27 July 2020; Accepted: 21 January 2021;

Published online: 25 February 2021

\section{References}

1. Guan, D. et al. Global supply-chain effects of COVID-19 control measures. Nat. Hum. Behav. 4, 577-587 (2020).

2. Hallegatte, S. An adaptive regional input-output model and its application to the assessment of the economic cost of Katrina. Risk Anal. 28, 779-799 (2008).

3. Hallegatte, S. Modeling the role of inventories and heterogeneity in the assessment of the economic costs of natural disasters. Risk Anal. 34, 152-167 (2014).

4. Aguiar, A., Chepeliev, M., Corong, E. L., McDougall, R. \& van der Mensbrugghe, D. The GTAP Data Base: version 10. J. Glob. Econ. Anal. 4, $1-27$ (2019).

5. Verschuur, J., Koks, E. E. \& Hall, J. W. Observed impacts of the COVID-19 pandemic on global trade. Nat. Hum. Behav. https://doi.org/10.1038/ s41562-021-01060-5 (2021).

6. Saghir, J. \& Hoekman, B. Air Freight: A Market Study with Implications for Landlocked Countries (World Bank, 2009).

7. COVID-19 and International Trade: Issues and Actions (OECD, 2020); http://www.oecd.org/coronavirus/policy-responses/covid-19-and-internat ional-trade-issues-and-actions-494da2fa/

8. Coronavirus Disease 2019 (COVID-19) Update (Kuehne+Nagel, 2020); https://home.kuehne-nagel.com/-/knowledge/updates-corona-virus-covid-19 (accessed December 20, 2020)

9. Navigating the Impact of COVID-19 (1010DATA, 2020); https://1010data.com/ covid-19/\#

10. Statistics of Main Exports by Month (General Department of Vietnam Customs, 2020); https://www.customs.gov.vn/Lists/EnglishStatisticsCalendars/ Attachments/1110/2020-T06T-2X(EN-PR).pdf

11. Statistics of Main Imports by Month (General Department of Vietnam Customs, 2020); https://www.customs.gov.vn/Lists/EnglishStatisticsCalendars/ Attachments/1111/2020-T06T-2N(EN-PR).pdf

\section{Acknowledgements}

D.G. acknowledges support from the National Natural Science Foundation of China (72091514).

\section{Author contributions}

D.W., K.H., X.L., D.M.C., S.H. and D.G. contributed equally to the manuscript and all drafted parts of this reply. All authors approved the final version.

\section{Competing interests}

The authors declare no competing interests.

\section{Additional information}

Correspondence and requests for materials should be addressed to D.G.

Peer review information Nature Human Behaviour thanks the anonymous reviewers for their contribution to the peer review of this work.

Reprints and permissions information is available at www.nature.com/reprints.

Publisher's note Springer Nature remains neutral with regard to jurisdictional claims in published maps and institutional affiliations.

(c) The Author(s), under exclusive licence to Springer Nature Limited 2021 